ARTICLE

\title{
Observation of strongly entangled photon pairs from a nanowire quantum dot
}

Marijn A.M. Versteegh ${ }^{1, \star}$, Michael E. Reimer ${ }^{1, \star}$, Klaus D. Jöns $^{1, \star}$, Dan Dalacu ${ }^{2}$, Philip J. Poole ${ }^{2}$, Angelo Gulinatti ${ }^{3}$, Andrea Giudice ${ }^{4} \&$ Val Zwiller ${ }^{1}$

A bright photon source that combines high-fidelity entanglement, on-demand generation, high extraction efficiency, directional and coherent emission, as well as position control at the nanoscale is required for implementing ambitious schemes in quantum information processing, such as that of a quantum repeater. Still, all of these properties have not yet been achieved in a single device. Semiconductor quantum dots embedded in nanowire waveguides potentially satisfy all of these requirements; however, although theoretically predicted, entanglement has not yet been demonstrated for a nanowire quantum dot. Here, we demonstrate a bright and coherent source of strongly entangled photon pairs from a positioncontrolled nanowire quantum dot with a fidelity as high as $0.859 \pm 0.006$ and concurrence of $0.80 \pm 0.02$. The two-photon quantum state is modified via the nanowire shape. Our new nanoscale entangled photon source can be integrated at desired positions in a quantum photonic circuit, single-electron devices and light-emitting diodes.

\footnotetext{
${ }^{1}$ Quantum Transport, Kavli Institute of Nanoscience, Delft University of Technology, Lorentzweg 1, 2628CJ Delft, The Netherlands. ${ }^{2}$ National Research Council of Canada, Ottawa, Ontario, Canada K1A OR6. ${ }^{3}$ Politecnico di Milano, Dipartimento di Elettronica Informazione e Bioingegneria, piazza Leonardo da Vinci 32, 20133 Milano, Italy. ${ }^{4}$ Micro Photon Devices, Via Stradivari 4, 39100 Bolzano, Italy. * These authors contributed equally to this work.

Correspondence and requests for materials should be addressed to M.A.M.V. (email: marijn.versteegh@univie.ac.at).
} 
T here are demanding requirements for an 'ideal' entangledphoton source for implementing ambitious schemes in quantum information processing, such as that of a quantum repeater ${ }^{1}$. The source should meet the following criteria: high brightness combined with high-fidelity entanglement $^{2}$, on-demand generation ${ }^{3}$, high extraction efficiency $^{4}$, directional ${ }^{5}$ and coherent emission ${ }^{6}$, as well as position control at the nanoscale ${ }^{7}$. It is extremely difficult to meet all of these requirements in a single device. Good candidates are semiconductor quantum dots embedded in nanowires.

The high refractive index of a nanowire waveguide around a quantum dot ensures that the emitted light is guided in the desired direction and a tapered end makes the light extraction very efficient ${ }^{4}$. With such a design, efficient single-photon generation has been demonstrated from a single nanowire quantum $\operatorname{dot}^{5}$. In addition, the emission mode-profile was shown to be directional and Gaussian ${ }^{8,9}$, a key requirement for efficient long-distance quantum communication in wellestablished telecommunication technology. Nanowires can be controllably positioned in uniform arrays ${ }^{10,11}$, with the ability to independently control the dot size and waveguide shell around $i^{12}{ }^{12}$. Silicon segments and substrates can be included in the design ${ }^{13-16}$ and electrical contacts have been demonstrated on single nanowires for single-electron devices ${ }^{17}$, light-emitting diodes $^{18}$, as well as single-photon avalanche photodiodes ${ }^{19}$. A significant advantage of using nanowire waveguides for efficient light extraction over other existing approaches, such as optical microcavities ${ }^{20}$, is the broad frequency bandwidth of operation ${ }^{21}$, which is needed for achieving bright entangled photon-pair generation via the biexciton-exciton radiative cascade. This approach is especially advantageous for quantum dots emitting over a large spectral range and may also be implemented with II-VI quantum dots where the biexciton binding energy is very large $(>20 \mathrm{meV})^{22}$.

A key feature of nanowires with embedded quantum dots grown in the [111]-direction is that the fine-structure splitting is expected to vanish ${ }^{23}$, which should result in excellent entangled photon emission via the biexciton-exciton radiative cascade ${ }^{24}$. Our measurements realize this prediction and demonstrate the generation of strongly entangled photon pairs for the first time from a nanowire quantum dot. Our sources are ready to implement in advanced quantum information processing schemes without the need for any post-growth manipulation ${ }^{25}$ or temporal post-selection ${ }^{26}$. Temporal post-selection can be a major source of photon losses and puts additional requirements on the measurement, thus limiting the scalability of quantum dotbased entangled photon sources. For practical applications it is therefore very useful that we can avoid temporal post-selection. Finally, due to the efficient waveguiding and the tapered end, which we created during the bottom-up growth of the nanowire, we measure a light extraction efficiency of $18 \pm 3 \%$ for the source. Importantly, due to a recent breakthrough in the nanowire growth ${ }^{12}$, this high efficiency is obtained while potentially meeting all of the criteria of an ideal entangled photon source.

\section{Results}

Site-controlled quantum dots in tapered nanowire waveguides. The nanowires were grown by selective-area chemical beam epitaxy, which allows for control of the dot size and position, as well as enabling growth of the waveguide shell around the dot for efficient light extraction (see Methods). This technique has been demonstrated to yield defect-free, pure wurtzite nanowires, which is essential to obtain long single-photon coherence ${ }^{27}$. Figure 1a shows a scanning electron microscopy (SEM) image of a tapered InP nanowire waveguide containing an InAsP segment, $200 \mathrm{~nm}$ from the nanowire base, defining the optically active quantum dot that we study.

A spectrum taken under the excitation condition used for the quantum-state tomography measurements is depicted in Fig. 1b. By performing cross-correlation measurements ${ }^{28}$, shown in the inset, and power-dependent measurements (see Supplementary Fig. 1), we identified the biexciton $(\mathrm{XX})$ and exciton $\left(\mathrm{X}_{\mathrm{A}}\right.$ and $\left.\mathrm{X}_{\mathrm{B}}\right)$ transitions. The $\mathrm{XX}-\mathrm{X}_{\mathrm{B}}$ cascade produces entangled photons. In contrast, a weak cascade is observed for $\mathrm{XX}-\mathrm{X}_{\mathrm{A}}$, which does not show entanglement. From these observations, $X_{A}$ could be either a charged exciton or an exciton with a different hole state than $\mathrm{X}_{\mathrm{B}}$ as is permitted by the wurtzite crystal structure. The transitions XX and $\mathrm{X}_{\mathrm{B}}$ are resolution limited; single-photon interference measurements show excellent coherence of our entangled photons (see Supplementary Fig. 2 and Supplementary Note 1). Autocorrelation measurements at saturation of the $\mathrm{XX}$ and $\mathrm{X}_{\mathrm{B}}$ transitions show strong antibunching, indicative of nearly perfect single-photon pairs from the $\mathrm{XX}-\mathrm{X}_{\mathrm{B}}$ cascade (see Supplementary Fig. 3).

Light extraction efficiency. From the single-photon detector counts, 55 kilocounts per second for $\mathrm{XX}$ and 15 kilocounts per second for $\mathrm{X}_{\mathrm{B}}$, under pulsed excitation at $80 \mathrm{MHz}$, we calculate a collection of 7.9 million $\mathrm{XX}$ and 2.0 million $\mathrm{X}_{\mathrm{B}}$ photons per second into the first objective when taking our $0.7 \pm 0.1 \%$ setup efficiency at $\sim 900 \mathrm{~nm}$ into account. The $\mathrm{X}_{\mathrm{A}}$ transition shows the highest intensity of 12.1 million photons per second and saturates our spectrometer's CCD camera with integration times as short as $1 \mathrm{~s}$ under the excitation conditions used throughout our study (Fig. 1b). This radiative recombination pathway from XX competes strongly with the $\mathrm{X}_{\mathrm{B}}$ emission, thus reducing the entangled photon-pair generation efficiency. Taking into account this competing recombination pathway for $\mathrm{XX}$, we calculate a light extraction efficiency of $18 \pm 3 \%$ for the source. We expect that adding a gold mirror with thin dielectric layer below the nanowire will boost the efficiency nearly twofold 21 . Combining this mirror with further engineering of the nanowire shape promises extraction efficiencies exceeding 90\% (ref. 29).

Low fine-structure-splitting system. Using polarization-dependent measurements, presented in Fig. 1c, we obtain an estimation for the excitonic fine-structure splitting, $S$, by subtracting the XX transition from the $X_{B}$ transition energy ${ }^{30}$. We obtain from the sine-function fit a fine-structure splitting of $1.2 \mu \mathrm{eV}$. In the case of nanowires, the small fine-structure splitting is a result of growth on a [111]-oriented substrate and the symmetric hexagonal crosssection of the nanowire core, defining the quantum dot. This small value that we measure for the fine-structure splitting is crucial for the entanglement observation between $\mathrm{XX}$ and $\mathrm{X}_{\mathrm{B}}$ photons without any temporal post-selection and is representative of the sample where, remarkably, a high percentage $(>50 \%)$ of the measured quantum dots show a fine-structure splitting below $2 \mu \mathrm{eV}$ (Supplementary Fig. 4). The period of precession of the $X_{B}$ spin can be estimated as $h / S=3.5 \mathrm{~ns}$ (ref. 31), where $h$ is Planck's constant. This period of precession is much longer than the $X_{B}$ lifetime of $0.50 \pm 0.01 \mathrm{~ns}$ as extracted from the $\mathrm{XX}-\mathrm{X}_{\mathrm{B}}$ cross-correlation measurements without polarization selection (Supplementary Fig. 5). Therefore, the $X_{B}$ spin precession has only little influence on the correlations in polarization between the two photons.

Polarization-entangled photon pairs. Twelve cross-correlation measurements in the rectilinear, diagonal and circular polarization bases are shown in Fig. 2, where each histogram is composed of $64 \mathrm{ps}$ time bins. In the correlation measurements, the first letter stands for the measured polarization of the XX photon, whereas 
a

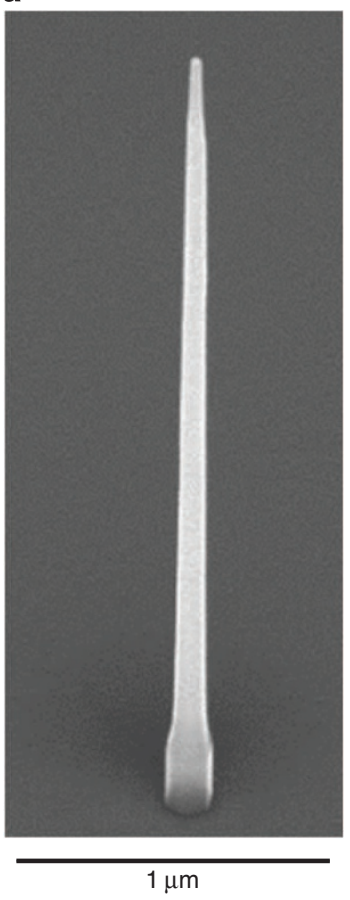

b
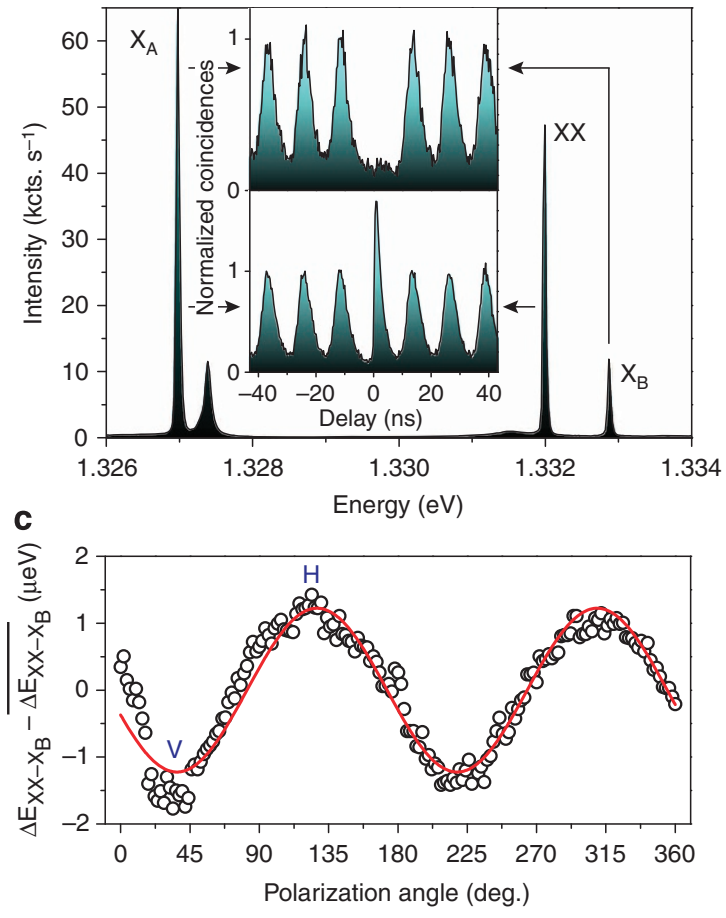

Figure 1 | Nanowire quantum dot sample. (a) Scanning electron microscopy image of a tapered nanowire waveguide with embedded quantum dot. (b) Photoluminescence spectrum of a single InAsP quantum dot embedded in an InP nanowire. The spectrum was taken at the excitation power used for the cross-correlation measurements needed to reconstruct the density matrix (100 nW), which is close to saturation of both $X X$ and $X_{B}$ transitions. Note that the excitonic transition $X_{A}$ saturates the CCD camera. (c) Polarization-dependent measurement to determine the excitonic fine-structure splitting. To increase the accuracy of the polarization measurement we plot the relative difference between biexciton $X X$ and exciton $X_{B}$ emission energy. The amplitude of the sine-function fit indicates a fine-structure splitting of $1.2 \mu \mathrm{eV}$.

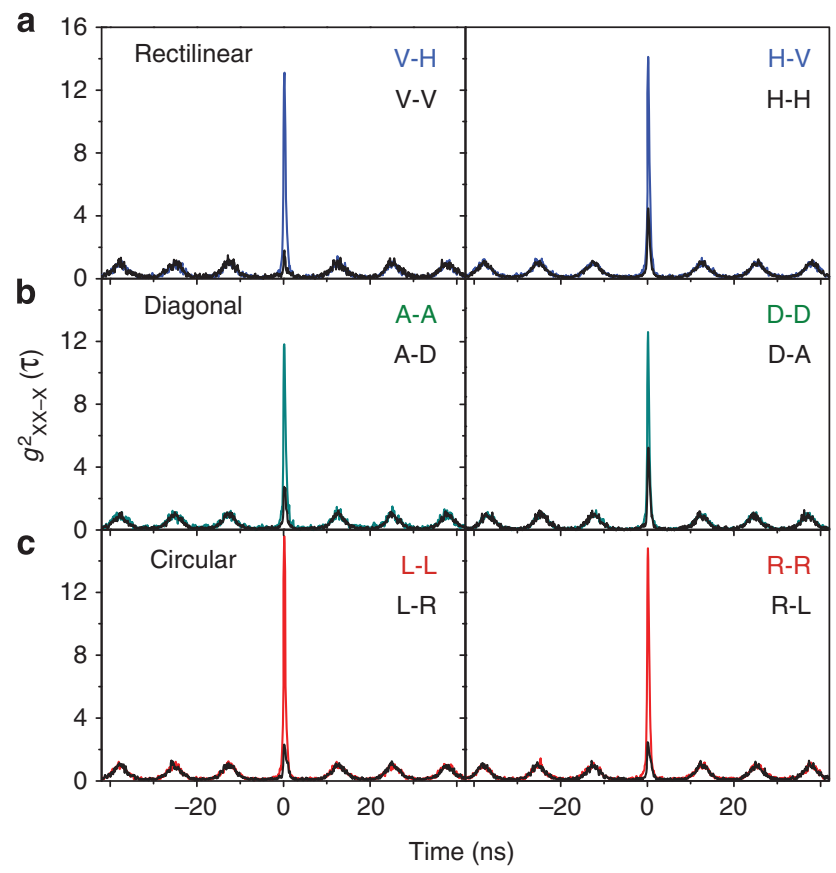

Figure 2 | Cross-correlation measurements for the three different bases: (a) Rectilinear, (b) diagonal and (c) circular. The plotted data are normalized to the Poisson level of the side peaks. Start: biexciton; stop: exciton $X_{B}$. The first letter stands for the measured polarization of the biexciton photon, whereas the second letter stands for the measured polarization of the exciton photon. the second letter stands for the $\mathrm{X}_{\mathrm{B}}$ photon. The strong correlations in $H V, V H, D D, A A, R R$ and $L L$, together with the weak correlations in $H H, V V, D A, A D, R L$ and $L R$, show that the two photons from the $\mathrm{XX}-\mathrm{X}_{\mathrm{B}}$ cascade are entangled. Here, $H$ and $V$ are orthogonal linear polarizations (horizontal and vertical), $D=(H+V) / \sqrt{2}$ and $A=(H-V) / \sqrt{2}$ are diagonal and antidiagonal linear polarizations, whereas $R=(H+i V) / \sqrt{2}$ and $L=(H-i V) / \sqrt{2}$ are righthanded and lefthanded circular polarizations.

The quantum state we observe is different from the state that is measured for self-assembled quantum dots $7,25,32-35$. Typically, one measures for the $\mathrm{XX}-\mathrm{X}$ cascade bunching (positive correlations) in $H H$ and $R L$, and antibunching (negative correlations) in $H V$ and $R R$. However, we observe the opposite (Fig. 2a,c). Only in the diagonal basis we see the usual correlations: bunching in $D D$ and antibunching in $D A$. These results show that the two-photon quantum state is closer to $(|H V\rangle+|V H\rangle) / \sqrt{ } 2$ than to the commonly measured state $(|H H\rangle+|V V\rangle) / \sqrt{ } 2$ (refs $7,25,32-35$ ).

Quantum-state tomography. We performed quantum-state tomography ${ }^{36}$ to determine more precisely the quantum state of the photons and the degree of entanglement. The raw crosscorrelation measurements needed to reconstruct the density matrix are shown in Supplementary Fig. 6. The resulting density matrix is given in Fig. 3a,b. The concurrence is $0.57 \pm 0.02$. A positive value for the concurrence means that the correlations cannot be explained classically and that the photons are quantum entangled. In this calculation, all correlation counts in the full time window of $6.02 \mathrm{~ns}$ are taken into account. The two-photon 

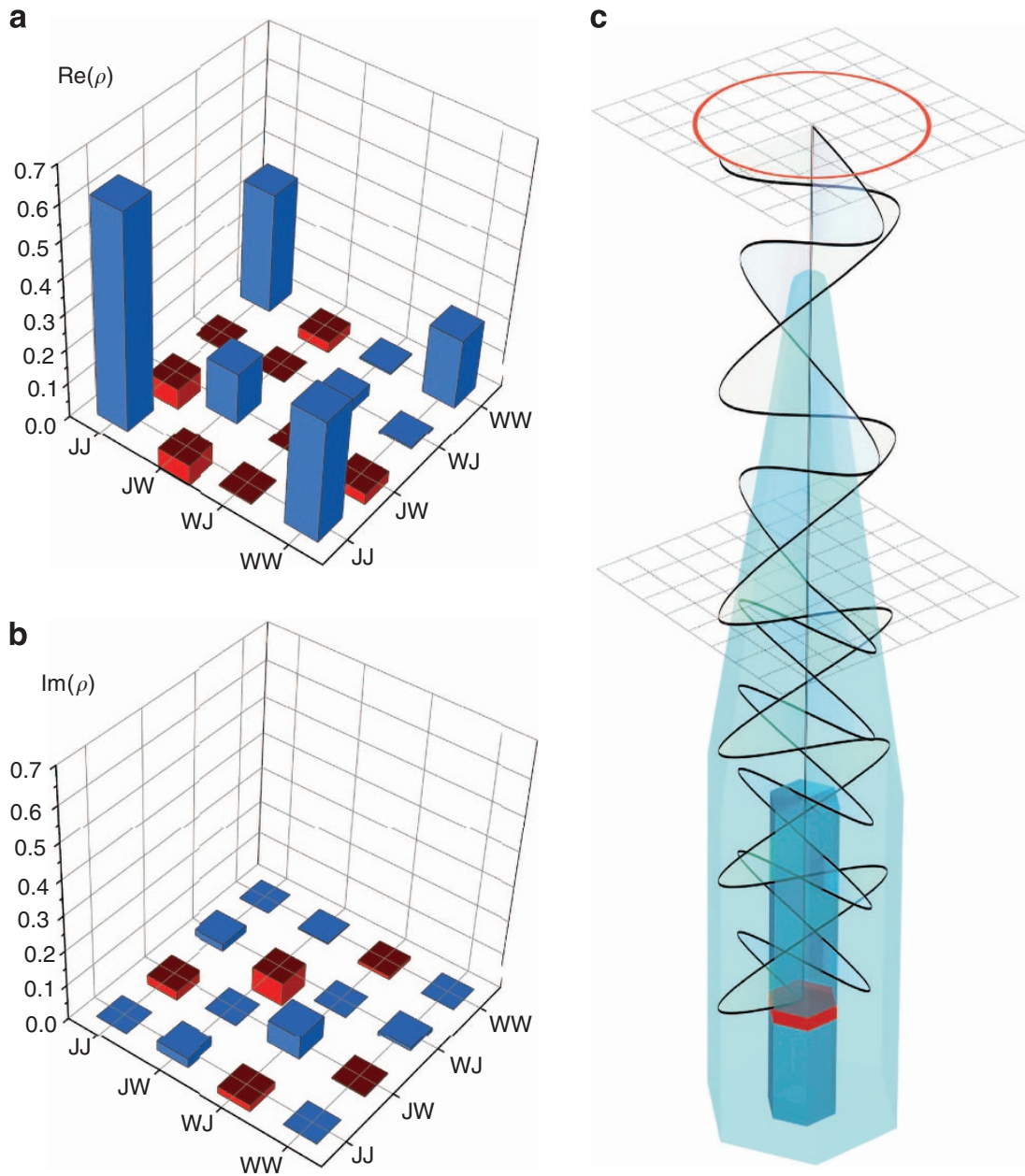

Figure 3 | Quantum-state tomography. Real (a) and imaginary part (b) of the density matrix for the full time window of $6 \mathrm{~ns}$, in the rotated basis. The positive matrix elements are blue, and the negative matrix elements are red. (c) Illustration of the effect of birefringence in the nanowire. The orthogonal waves inside the nanowire experience different refractive indices, and therefore their wavelengths inside the waveguide are unequal. As a result, the polarization of the light emission by the quantum dot (red) is modified leading to a different quantum state. The tapered section of the nanowire is more symmetric and is free of birefringence.

Table 1 | Calculated concurrences and the fidelities for five different time windows.

\begin{tabular}{lccc} 
Time window (\% of coincidence counts) & Concurrence & Fidelity to $(|\mathrm{J}\rangle+\mid \mathrm{WW}>) / \sqrt{2}$ & Fidelity to general maximally entangled state \\
\hline $6.02 \mathrm{~ns}(100)$ & $0.57 \pm 0.02$ & $0.762 \pm 0.002\left(\alpha=33^{\circ}, \beta=46^{\circ}\right)$ & $0.765 \pm 0.002$ \\
$1.40 \mathrm{~ns}(80)$ & $0.64 \pm 0.02$ & $0.793 \pm 0.002\left(\alpha=33^{\circ}, \beta=47^{\circ}\right)$ & $0.796 \pm 0.002$ \\
$0.70 \mathrm{~ns}(59)$ & $0.69 \pm 0.02$ & $0.814 \pm 0.003\left(\alpha=34^{\circ}, \beta=48^{\circ}\right)$ & $0.818 \pm 0.003$ \\
$0.38 \mathrm{~ns}(35)$ & $0.73 \pm 0.02$ & $0.839 \pm 0.003\left(\alpha=35^{\circ}, \beta=49^{\circ}\right)$ & $0.842 \pm 0.003$ \\
$0.13 \mathrm{~ns}(10)$ & $0.80 \pm 0.02$ & $0.854 \pm 0.006\left(\alpha=36^{\circ}, \beta=50^{\circ}\right)$ & $0.859 \pm 0.006$
\end{tabular}

The fraction of the correlation events taken into account for a certain time window are given in brackets in the first column.

state has a fidelity of $0.762 \pm 0.002$ to the maximally entangled state $(|J\rangle\rangle+|W W\rangle) / \sqrt{ } 2$, where $J=H e^{-i \beta} \cos \alpha+V e^{-i \beta} \sin \alpha$ and $W=-H e^{i \beta} \sin \alpha+V e^{i \beta} \cos \alpha$ are two orthogonal elliptical polarizations. The angles $\alpha$ and $\beta$ are specified in Table 1 . The classical limit is 0.5 , so this result shows a strong degree of entanglement, even without temporal selection.

Selection of a narrower time window yields higher values for the concurrence and the fidelity (Table 1). For example, for a time window of $0.13 \mathrm{~ns}$ we calculate a concurrence of $0.80 \pm 0.02$ and a fidelity of $0.854 \pm 0.006$. The density matrix for this time window is presented in Supplementary Fig. 7. Temporal selection yields stronger entanglement, because within a narrow time window the effects of spin precession and dephasing processes are smaller ${ }^{31}$. When we do not restrict our analysis to states of the form $(|J J\rangle+|W W\rangle) / \sqrt{ } 2$, but instead calculate the fidelity to a general maximally entangled two-photon state, we find only slightly higher values (Table 1). The maximally entangled states to which the fidelity is maximal are very close to states of the form $(|J J\rangle+|W W\rangle) / \sqrt{ } 2$

Two-photon quantum state modified by birefringence. Why do we measure $(|J J\rangle+|W W\rangle) / \sqrt{ } 2$ and not the usual twophoton state that is measured for quantum dots, namely 
a
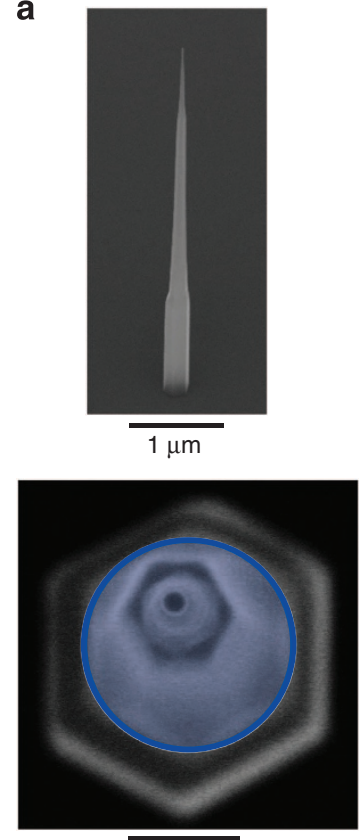

$100 \mathrm{~nm}$ b
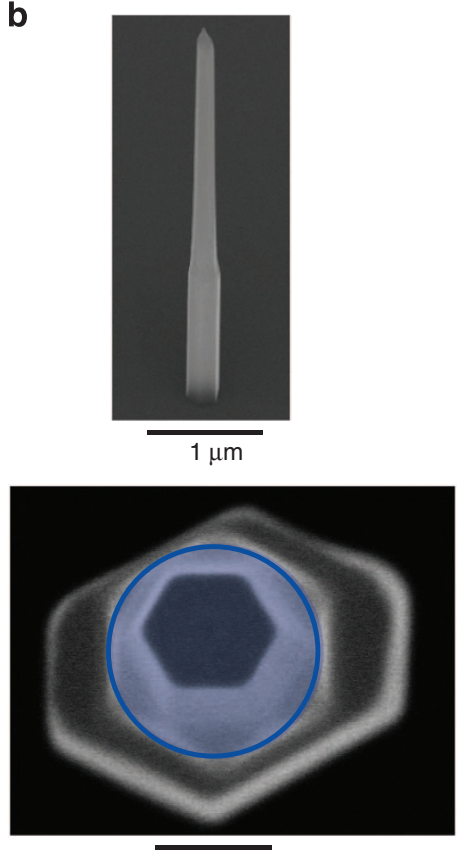

$100 \mathrm{~nm}$
Figure 4 | Nanowire birefringence. SEM images of: (a) symmetric nanowire waveguide, and (b) asymmetric nanowire waveguide. Top panel: side-view SEM images of nanowires with a tilt angle of 45 degrees. Bottom panel: SEM images of the nanowires viewed from the top at a small tilt angle. The blue-shaded circle represents the opening in the $\mathrm{SiO} 2$ mask. The example of the nanowire elongation in $\mathbf{b}$ is an extreme example that leads to geometric birefringence and corresponding rotation of the quantum state.

$(|H H\rangle+|V V\rangle) / \sqrt{ } 2$ ? The most probable reason is that the nanowire has a small anisotropy: it could have a slightly elongated cross-section. An extreme case is shown in the SEM image of Fig. 4b. Such an anisotropy may be formed during the growth of the cladding around the core, and would then be unrelated to the shape of the quantum dot (for details of the growth, see the Methods section). As a comparison, we show a symmetric nanowire waveguide in the SEM image of Fig. 4a. In case of an elongated cross-section the effective refractive indices are different for the polarizations along the short and the long axis of the nanowire. Here, we could imagine that the quantum dot emits photon pairs in the usual entangled quantum state $(|H H\rangle+|V V\rangle) / \sqrt{ } 2$. As the emitted photons are guided along the nanowire, the two-photon state is modified by birefringence into $(|J J\rangle+|W W\rangle) / \sqrt{ } 2$, as is illustrated in Fig. 3c. Thus, $H H$ and $V V$ correlations rotate into predominantly $R R$ and $L L$ correlations, while $R L$ and $L R$ turn mostly into $H V$ and $V H$, which explains the observations of Fig. 2. For a nanowire waveguide of $6 \mu \mathrm{m}$ length a difference of effective refractive index of order 0.1 would be enough to explain the magnitude of the observed rotation. Apart from birefringence in the waveguide, the polarization state of the emitted photons could also have been influenced by $\Gamma_{7}$ and $\Gamma_{9}$ hole mixing in the wurtzite quantum dot.

\section{Discussion}

In summary, we used a wurtzite nanowire quantum dot to generate single pairs of polarization-entangled photons with a fidelity as high as $0.859 \pm 0.006$ and a concurrence up to $0.80 \pm 0.02$. Furthermore, a high degree of entanglement is maintained (fidelity of $0.762 \pm 0.002$ ) without any temporal post-selection. This first observation of entangled photon-pair generation from a nanowire quantum dot, which combines the desired properties of an ideal entangled photon source, opens new opportunities in quantum optics, integrated quantum photonic circuits $^{37,38}$ and quantum information processing.

To realize an ideal entangled photon source in future work there are several properties of our source to consider. First, quantum dot-entangled photon sources have not yet reached the fidelity or concurrence values of parametric down-conversion sources ${ }^{2,39}$. However, with recently available post-growth tuning methods to bring the fine-structure splitting of almost any quantum dot near zero $^{25}$ and two-photon resonant excitation ${ }^{3}$, the fidelity of these quantum dot sources are approaching that of parametric downconversion sources. Second, the single-photon coherence of the emitted photon pairs is not yet Fourier-transform limited, which is needed for advanced quantum information-processing schemes. Such Fourier-transform-limited photons may be reached by combining two-photon resonant excitation techniques ${ }^{3,40}$, cooling of the quantum dot sample to $300 \mathrm{mK}^{28}$ and by accelerating the quantum dot emission via the Purcell effect ${ }^{20}$. Finally, the major advantage of tapered nanowire waveguides over other approaches is the light extraction efficiency, which promises entangled photon-pair extraction efficiencies exceeding $90 \%$ due to the broadband frequency of operation ${ }^{29}$. Such efficiencies would surpass the state-of-the-art entangled photon-pair efficiency of $12 \%{ }^{20}$, without the stringent requirements needed to engineer both the exciton and biexciton into resonance with a cavity mode by using post-growth manipulation of pre-selected quantum dots.

Note: Similar work is reported by Huber et al. ${ }^{26}$

\section{Methods}

Nanowire quantum dot growth. The InP nanowires containing single InAsP quantum dots were grown using chemical beam epitaxy with trimethylindium and pre-cracked $\mathrm{PH}_{3}$ and $\mathrm{AsH}_{3}$ sources. The nanowires were grown on a $\mathrm{SiO}_{2}$ patterned (111)B InP substrate consisting of circular holes opened up in the oxide mask using electron-beam lithography and a hydrofluoric acid wet-etch. Au was deposited in these holes using a self-aligned lift-off process, which allows the nanowires to be positioned at known locations on the substrate ${ }^{41}$. The thickness of the deposited gold is chosen to give $20-\mathrm{nm}$ to $40-\mathrm{nm}$ diameter particles, depending on the size of the hole opening. The nanowires were grown at $420^{\circ} \mathrm{C}$ with a trimethylindium flux equivalent to that used for a planar InP growth rate of $0.1 \mu \mathrm{m} \mathrm{h}^{-1}$ on (001) InP substrates at a temperature of $500^{\circ} \mathrm{C}$. The growth is a two-step process: (i) growth of a nanowire core containing the quantum dot, nominally $200 \mathrm{~nm}$ from the nanowire base, and (ii) cladding of the core to realize nanowire diameters for efficient light extraction (around $200 \mathrm{~nm}$ ). The quantum dot diameters are determined by the size of the nanowire core. In this study, we investigated quantum dot diameters ranging from $\sim 25$ to $30 \mathrm{~nm}$.

The nanowire core was grown for $26 \mathrm{~min}$ at a $\mathrm{PH}_{3}$ flow of 3 s.c.c.m. The dot was incorporated by switching from a $\mathrm{PH}_{3}$ to an $\mathrm{AsH}_{3}$ overpressure for $3 \mathrm{~s}$ after $15 \mathrm{~min}$ of growth. This growth time results in a quantum dot height of $\sim 6 \mathrm{~nm}$ as determined in our previous studies ${ }^{12}$, using an energy-dispersive $\mathrm{X}$-ray spectroscopy line scan along the nanowire, for a sample with nominally identical growth conditions. We note that our quantum dots are grown during $3 \mathrm{~s}$, resulting in taller quantum dots with longer emission wavelength, as compared with the work of Huber et al. ${ }^{26}$ who used a growth time of $2 \mathrm{~s}$. Our growth conditions result in very small fine-structure splittings as shown in Supplementary Fig. 4.

The nanowire cladding was grown by increasing the $\mathrm{PH}_{3}$ flow to 9 s.c.c.m. The total growth time was $120 \mathrm{~min}$. To realize the smooth tapering towards the tip, the nanowire was made longer than the diffusion length of indium. Most nanowires, including the quantum dots, have a pure wurtzite crystal structure. The nanowire axis is the wurtzite $c$ axis.

Nanowire waveguide elongation. Radial growth is nominally constrained by the oxide opening, and the nanowire cross-section has a hexagonal symmetry. Optimal coupling of the quantum dot emission to the waveguide mode requires diameters exceeding that of the oxide opening. This is achieved by increasing the cladding growth time, which results in the nanowire overgrowing the oxide opening. No longer constrained by the opening, the hexagonal symmetry may be distorted (see Fig. 4). This asymmetry results in a geometric birefringence and concomitant rotation of the polarization state emitted by the quantum dot.

Optical measurements. The optical measurements were performed in a standard confocal microscopy setup where the quantum dot sample is cooled to a temperature of $\mathrm{T}=5 \mathrm{~K}$ in a closed-cycle cryostat. The setup consists of two spectrometers both equipped with red-enhanced single-photon avalanche diodes having 
75 ps time resolution, dark count rates as low as 80 counts per second and quantum efficiency of $11.5 \%$ at $930 \mathrm{~nm}^{42}$. A set of waveplates and polarizers placed in front of each spectrometer was used to perform polarization-dependent cross-correlation measurements. One spectrometer is set to the biexciton (XX) transition and the other to the exciton $\left(\mathrm{X}_{\mathrm{B}}\right)$ transition. Each correlation measurement was done with $6,000 \mathrm{~s}$ of integration to reach over 1,000 correlations in each side peak. For all photoluminescence and correlation measurements, we use a Ti:Sapphire laser emitting 3-ps-long pulses at $750 \mathrm{~nm}$ with a repetition rate of $80 \mathrm{MHz}$ to excite the quantum dot.

\section{References}

1. Briegel, H.-J., Dür, W., Cirac, J. I. \& Zoller, P. Quantum repeaters: the role of imperfect local operations in quantum communication. Phys. Rev. Lett. 81, 5932-5935 (1998).

2. Kwiat, P. G., Waks, E., White, A. G., Appelbaum, I. \& Eberhard, P. H Ultrabright source of polarization-entangled photons. Phys. Rev. A 60, R773-R776 (1999).

3. Müller, M., Bounouar, S., Jöns, K. D., Glässl, M. \& Michler, P. On-demand generation of indistinguishable polarization-entangled photon pairs. Nat. Photon. 8, 224-228 (2014).

4. Claudon, J. et al. A highly efficient single-photon source based on a quantum dot in a photonic nanowire. Nat. Photon. 4, 174-177 (2010)

5. Reimer, M. E. et al. Bright single-photon sources in bottom-up tailored nanowires. Nat. Commun. 3, 737 (2012).

6. Ates, S. et al. Post-selected indistinguishable photons from the resonance fluorescence of a single quantum dot in a microcavity. Phys. Rev. Lett. 103, 167402 (2009).

7. Juska, G., Dimastrodonato, V., Mereni, L. O., Gocalinska, A. \& Pelucchi, E. Towards quantum-dot arrays of entangled photon emitters. Nat. Photon. 7, 527-531 (2013).

8. Munsch, M. et al. Erratum: dielectric GaAs antenna ensuring an efficient broadband coupling between an InAs quantum dot and a Gaussian optical beam. Phys. Rev. Lett. 111, 239902 (2013).

9. Bulgarini, G. et al. Nanowire waveguides launching single photons in a Gaussian mode for ideal fiber coupling. Nano Lett. 14, 4102-4106 (2014).

10. Borgström, M. T., Immink, G., Ketelaars, B., Algra, R. \& Bakkers, E. P. A. M. Synergetic nanowire growth. Nat. Nanotechnol. 2, 541-544 (2007).

11. Dorenbos, S. N. et al. Position controlled nanowires for infrared single photon emission. Appl. Phys. Lett. 97, 171106 (2010).

12. Dalacu, D. et al. Ultraclean emission from InAsP quantum dots in defect-free wurtzite InP nanowires. Nano Lett. 12, 5919-5923 (2012).

13. Hertenberger, S. et al. Growth kinetics in position-controlled and catalyst-free InAs nanowire arrays on $\mathrm{Si}(111)$ grown by selective area molecular beam epitaxy. J. Appl. Phys. 108, 114316 (2010).

14. Kang, J.-H. et al. Defect-free Gaas/AlGaAs core-shell nanowires on $\mathrm{Si}$ substrates. Cryst. Growth Des. 11, 3109-3114 (2011).

15. Hocevar, M. et al. Growth and optical properties of axial hybrid III-V/silicon nanowires. Nat. Commun. 3, 1266 (2012).

16. Munshi, A. M. et al. Position-controlled uniform GaAs nanowires on silicon using nanoimprint lithography. Nano Lett. 14, 960-966 (2014).

17. Reimer, M. E. et al. Electric field induced removal of the biexciton binding energy in a single quantum dot. Nano Lett. 11, 645-650 (2011).

18. Minot, E. D. et al. Single quantum dot nanowire LEDs. Nano Lett. 7, 367-371 (2007).

19. Bulgarini, G. et al. Avalanche amplification of a single exciton in a semiconductor nanowire. Nat. Photon. 6, 455-458 (2012).

20. Dousse, A. et al. Ultrabright source of entangled photon pairs. Nature 466, 217-220 (2010)

21. Bleuse, J. et al. Inhibition, enhancement, and control of spontaneous emission in photonic nanowires. Phys. Rev. Lett. 106, 103601 (2011).

22. Akimov, I. A., Andrews, J. T. \& Henneberger, F. Stimulated emission from the biexciton in a single self-assembled II-VI quantum dot. Phys. Rev. Lett. 96, 067401 (2006).

23. Singh, R. \& Bester, G. Nanowire quantum dots as an ideal source of entangled photon pairs. Phys. Rev. Lett. 103, 54 (2009).

24. Benson, O., Santori, C., Pelton, M. \& Yamamoto, Y. Regulated and entangled photons from a single quantum dot. Phys. Rev. Lett. 84, 2513-2516 (2000).

25. Trotta, R. et al. Highly entangled photons from hybrid piezoelectricsemiconductor quantum dot devices. Nano Lett. 14, 3439-3444 (2014).

26. Huber, T. et al. Polarization entangled photons from quantum dots embedded in nanowires. Preprint at http://arxiv.org/abs/1405.3765 (2014)
27. Reimer, M. E. et al. Overcoming power broadening of the quantum dot emission in a pure wurtzite nanowire. Preprint at http://arxiv.org/abs/ 1407.2833 (2014).

28. Baier, M. H, Malko, A., Pelucchi, E., Oberli, D. Y \& Kapon, E. Quantum-dot exciton dynamics probed by photon-correlation spectroscopy. Phys. Rev. B 73, 205321 (2006).

29. Friedler, I. et al. Solid-state single photon sources: the nanowire antenna. Opt. Expres 17, 2095-2110 (2009).

30. Young, R. J. et al. Bell-inequality violation with a triggered photon-pair source. Phys. Rev. Lett. 102, 030406 (2009).

31. Stevenson, R. M. et al. Evolution of entanglement between distinguishable light states. Phys. Rev. Lett. 101, 170501 (2008).

32. Akopian, N. et al. Entangled photon pairs from semiconductor quantum dots. Phys. Rev. Lett. 96, 130501 (2006).

33. Young, R. J. et al. Improved fidelity of triggered entangled photons from single quantum dots. New J. Phys. 8, 29 (2006)

34. Hafenbrak, R. et al. Triggered polarization-entangled photon pairs from a single quantum dot up to 30 K. New J. Phys. 9, 315 (2007).

35. Kuroda, T. et al. Symmetric quantum dots as efficient sources of highly entangled photons: Violation of Bell's inequality without spectral and temporal filtering. Phys. Rev. B 88, 041306(R) (2013).

36. James, D. F. V., Kwiat, P. G., Munro, W. J. \& White, A. G. Measurement of qubits. Phys. Rev. A 64, 052312 (2001).

37. Politi, A., Cryan, M. J., Rarity, J. G., Yu, S. \& O’Brien, J. L. Silica-on-silicon waveguide quantum circuits. Science 320, 646-649 (2008).

38. Silverstone, J. W. et al. On-chip quantum interference between silicon photonpair sources. Nat. Photon. 8, 104-108 (2014).

39. Fedrizzi, A., Herbst, T., Poppe, A., Jennewein, T. \& Zeilinger, A. A wavelengthtunable fiber-coupled source of narrowband entangled photons. Opt. Expres 15 , 15377-15386 (2007).

40. Jayakumar, H. et al. Deterministic photon pairs and coherent optical control of a single quantum dot. Phys. Rev. Lett. 110, 135505 (2013).

41. Dalacu, D. et al. Selective-area vapour-liquid-solid growth of InP nanowires. Nanotechnology 20, 395602 (2009).

42. Gulinatti, A. et al. New silicon SPAD technology for enhanced red-sensitivity, high-resolution timing and system integration. J. Modern Opt. 59, 1489-1499 (2012).

\section{Acknowledgements}

We acknowledge S. Höfling, T. Braun and R. Trotta for scientific discussions, L. Schweickert for the image illustrating the nanowire birefringence. This research was supported by the Dutch Foundation for Fundamental Research on Matter (FOM projectruimte 12PR2994), ERC, and the European Union Seventh Framework Programme 209 (FP7/2007-2013) under Grant Agreement No. 601126210 (HANAS).

\section{Author contributions}

M.E.R., M.A.M.V. and V.Z. conceived and designed the experiments. K.D.J., M.E.R. and M.A.M.V. performed the experiments. D.D. and P.J.P. fabricated the sample. M.A.M.V., M.E.R. and K.D.J. analysed the data. A.Gu. and A.Gi. developed the detectors. M.E.R., M.A.M.V., K.D.J. and V.Z. wrote the manuscript with input from the other authors.

\section{Additional information}

Supplementary Information accompanies this paper at http://www.nature.com/ naturecommunications

Competing financial interests: The authors declare no competing financial interests.

Reprints and permission information is available online at http://npg.nature.com/ reprintsandpermissions/

How to cite this article: Versteegh, M. A. M. et al. Observation of strongly entangled photon pairs from a nanowire quantum dot. Nat. Commun. 5:5298 doi: 10.1038/ncomms6298 (2014).

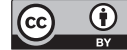

This work is licensed under a Creative Commons Attribution 4.0 International License. The images or other third party material in this article are included in the article's Creative Commons license, unless indicated otherwise in the credit line; if the material is not included under the Creative Commons license, users will need to obtain permission from the license holder to reproduce the material. To view a copy of this license, visit http://creativecommons.org/licenses/by/4.0/ 